\title{
Reexamination of Criticality Accident in JCO
}

\author{
Sachiko Oshima1, Miri Hisada², Yusuke Saito², Takehisa Fujita² \\ ${ }^{1}$ College of Industrial Technology, Nihon University, Narashino, Chiba, Japan \\ ${ }^{2}$ Department of Physics, Faculty of Science and Technology, Nihon University, Tokyo, Japan \\ Email: oshima.sachiko@nihon-u.ac.jp,fffujita@phys.cst.nihon-u.ac.jp
}

Received 22 June 2016; accepted 22 August 2016; published 25 August 2016

Copyright (C) 2016 by authors and Scientific Research Publishing Inc.

This work is licensed under the Creative Commons Attribution International License (CC BY). http://creativecommons.org/licenses/by/4.0/

(c) (i) Open Access

\begin{abstract}
Nuclear chain reactions are, by now, commonly used in the nuclear reactors, and thus it seems that there is no basic problem in fission processes from the scientific point of view. However, the criticality accident that occurred in JCO in 1999 suggests that one should carefully examine this accident from the nuclear physics point of view. Indeed the chain nuclear reactions should have taken place in the small area of space with $45 \mathrm{~cm}$ diameter disk times $30 \mathrm{~cm}$ height tank. In fact, when people carry the uranium nitrate solution into sedimentation tank, then this solution with uranium should get into the critical state at the $45 \ell$ of uranium nitrate solution. The root cause of the accident should not be very simple from the nuclear physics point, and it should be quite important to examine why the uranium nitrate solution with $45 \ell$ could have become critical.
\end{abstract}

\section{Keywords}

Nuclear Fission, Criticality, Mean Free Path

\section{Introduction}

The criticality accident that occurred in JCO in 1999 must be most serious, and it should not be very easy to understand why the nuclear chain reactions could proceed in a small area of space for a finite period of time. In this sense, it should be quite important to carry out the careful examination of criticality accidents from the nuclear physics point of view. It should be, of course, difficult to claim that the JCO accident can be a target of the scientific study since one cannot make the experimental study of the JCO type accidents. However, we believe that the basic mechanism of the criticality accident should be clarified why it could naturally occur in the small area of space.

This criticality accident occurred when workers in JCO company were carrying the uranium nitrate solution (18.8\% enriched uranium) into sedimentation tank [1]-[3]. Here, we should explain the working procedure 
which is taken by the workers in JCO. First, they make the uranium nitrate solution which is composed of $2.4 \mathrm{~kg}$ $\mathrm{U}_{3} \mathrm{O}_{8}$ with the nitric acid of $1.7 \ell$ in the stainless vessel. In addition, they add water to the uranium nitrate solution until the total volume becomes $6.5 \ell$. Then, they carry the $6.5 \ell$ solution into the sedimentation tank, and this working procedure is called one batch.

The criticality accident should have occurred in the middle of the seventh batch since the workers noticed blue lights that should be due to the Cherenkov radiation. In fact, two of the workers suffered from the neutron radiation.

A question should arise as to how the nuclear chain reactions could proceed within the small sedimentation tank (45 cm diameter, $60 \mathrm{~cm}$ high). There are, of course, some analysises of this criticality accident [4] [5]. However, these studies are mainly carried out for the computer simulation such that the total energy emitted via radiations can be reproduced in some way or the other. These investigations are, of course, very important in order to understand the accident cause. However, it is also important to carry out the study of the criticality accident from the nuclear physics point of view.

In this paper, we carry out careful calculations of the criticality accident in terms of the multiple scattering theory. Here, we want to understand why the nuclear chain reactions can proceed in the small area of space. In particular, we trace the nuclear fission reactions (nucleon-nucleon collision together with nuclear fission) each by each, and we clarify the microscopic processes why and how the criticality accident occurred. As a result, we should understand some specific reasons why the chain reactions can proceed, and this can be done by making use of the mean free path which is the result of the nuclear multiple scattering theory.

However, when we clarify how the criticality accident occurs, we face to the most difficult question as to why the criticality could stop. In this study, we find an answer for this question, though not necessarily sufficient. This mechanism of stopping criticality may be related to the quick settle of the uranium compound.

As a result of our calculation, we find a possible dangerous situation which was thought to be due to the 8th batch, if it were carried into the sedimentation tank. We see that the estimated energy release after the virtual 8th batch should become the same order of magnitude as the Chernobyl nuclear accident.

\section{Nuclear Chain Reactions}

Nuclear fission reaction by incident neutrons can be written as [6]

$$
n+{ }^{235} \mathrm{U} \rightarrow A_{1}+A_{2}+(2 \sim 3) n
$$

where $A_{1}, A_{2}$ are new nuclei which are produced in the reactions. In this reaction, there are two important points. The first one is concerned with two or three neutrons which are produced in the reactions. The second point is that the probability of this nuclear reactions is strongly based on the incident neutron energy, and the biggest cross section is for the incident neutron with almost zero energy (thermal energy).

The chain reactions indicate that the produced neutrons should be absorbed by another ${ }^{235} \mathrm{U}$ such that the nuclear fission can proceed further on. In addition, if the chain reactions continue to proceed without the aid of other external neutron sources, then this situation is called a criticality stage. In reactors, this criticality must be kept by controlling the number of neutrons involved in the chain reactions.

In normal reactors, a few \% enriched uranium should be commonly used, but in this JCO accident, 18.8\% enriched uranium were used, and this high enrichment should be one of the strong reasons why the nuclear reactions run wild.

\section{Why Criticality?}

Now, a question is as to why the criticality is realized in the small area of the sedimentation tank with $50 \ell$ of the uranium nitrate solution. That is, why nuclear chain reactions continue to occur in this small area. Here we clarify the basic mechanism of the criticality accident.

\subsection{Neutron Source}

The nuclear chain reactions should require thermal neutrons to start for the initial fission reactions. Since neutrons should decay within 15 minutes, they do not exist as a natural source. Neutrons should be produced in some way or the other. Here in this accident, the neutron source should be the decay of ${ }^{238} \mathrm{U}$ spontaneous 
fissions. The life time of ${ }^{238} \mathrm{U}$ is about 4.5 billion years and, in addition, the rate of the spontaneous fission to the total width is around $5.45 \times 10^{-7}$. Therefore, $1 \mathrm{~g}$ of ${ }^{238} \mathrm{U}$ make the spontaneous fission of 0.01 times per second. Since one batch contains $1.6 \mathrm{~kg}$ of ${ }^{238} \mathrm{U}$, we should find about 20 neutrons per second in the one batch solution.

\subsection{Mean Free Path of $\boldsymbol{n}$-235 $\mathrm{U}$ Fission (Fast Neutrons)}

The probability of nuclear fission of ${ }^{235} \mathrm{U}$ induced by neutrons should be evaluated in terms of mean free path of $\lambda$ inside the uranium nitrate solution. This mean free path of nuclear reactions can be obtained from the multiple scattering theory as

$$
\lambda=\frac{1}{\rho \sigma_{f}}
$$

This derivation of the mean free path (2) is based on the Glauber theory [7], and this theoretical frame work is well examined in atomic and nuclear reactions [8] [9]. Here, $\rho$ denotes the number density of ${ }^{235} \mathrm{U}$ in solution and $\sigma_{f}$ corresponds to the nuclear fission cross section of ${ }^{235} \mathrm{U}$ induced by neutrons. In fact, the number density of ${ }^{235} \mathrm{U}$ in one batch solution is $\rho \simeq 1.5 \times 10^{20} \mathrm{~cm}^{-3}$ which is a constant. On the other hand, the nuclear fission cross section $\sigma_{f}$ of ${ }^{235} \mathrm{U}$ induced by neutrons crucially depends on the incident energy of neutrons. The incident energy dependence of the observed cross sections $\sigma_{f}$ can be written as [10]

$$
\sigma_{f} \simeq\left\{\begin{array}{l}
585 \mathrm{~b}: E_{n} \simeq 0.025 \mathrm{eV} \\
1 \mathrm{~b}: E_{n} \simeq 1 \mathrm{MeV}
\end{array}\right.
$$

where $1 \mathrm{~b}=10^{-24} \mathrm{~cm}^{2}$.

\section{Mean Free Path of Prompt neutrons in Nuclear Fission}

In fission process, the average energy of prompt neutrons is around $1 \mathrm{MeV}$, and therefore the average mean free path of the prompt neutrons after fissions becomes

$$
\lambda_{f}=\frac{1}{\rho \sigma_{f}} \simeq 67 \mathrm{~m} .
$$

This is quite long in comparison with the scale of the tank, and therefore this prompt neutrons by themselves cannot induce subsequent fissions in corresponding solution in the tank. In this respect, we ask a question as to why the criticality should take place within the small sedimentation tank.

\section{Collision between Neutrons and Water Molecule}

In reality, the prompt neutrons may collide with protons in water molecule, and they should lose their energy by nucleon-nucleon collisions. Since the nuclear fission cross sections become largest for the thermal neutrons, the fission processes should start in case the prompt neutrons lose most of their energy inside the uranium nitrate solution.

\subsection{Energy Loss after the Collision of Prompt Neutrons with Protons in Water}

When the prompt neutron scatters with protons in water, this neutron should lose a half of its energy. This can be easily understood in the following way. First, we denote the incident momentum and energy of the neutron by $\boldsymbol{p}, E_{n}$ with $E_{n}=\frac{\boldsymbol{p}^{2}}{2 M}$, and the final momentum and energy by $\boldsymbol{k}, E_{n}^{\prime}$ with $E_{n}^{\prime}=\frac{\boldsymbol{k}^{2}}{2 M}$. In this case, we find an equation from the conservation law of momentum and energy as

$$
\frac{\boldsymbol{p}^{2}}{2 M}=\frac{\boldsymbol{k}^{2}}{2 M}+\frac{(\boldsymbol{p}-\boldsymbol{k})^{2}}{2 M}
$$

which can be solved and its solution becomes

$$
k=p \cos \theta .
$$

Since the observed scattering cross section does not depend on the scattering angles, we can make an average 
over the angles, and we obtain the average energy after the scattering

$$
E_{n}^{\prime}=\frac{1}{\pi} \int_{0}^{\pi} \frac{k^{2}}{2 M} \mathrm{~d} \theta=\frac{1}{\pi} \int_{0}^{\pi} \frac{p^{2}}{2 M} \cos ^{2} \theta \mathrm{d} \theta=\frac{1}{2} E_{n} .
$$

This means that a neutron should lose a half of its energy in each scattering process.

\subsection{The Mean Free Path of Neutrons Inside Water}

Now we calculate the mean free path of neutrons after the scattering with protons in one batch solution. The number density of protons in one batch solution is $\rho_{p} \simeq 4.9 \times 10^{22} \mathrm{~cm}^{-3}$. The neutron-proton cross section at low energy is observed as $\sigma_{n p} \simeq 20 \mathrm{~b}$ [11], and thus the mean free path of neutron in one batch solution becomes

$$
\lambda_{p}=\frac{1}{\rho_{p} \sigma_{n p}} \simeq 1 \mathrm{~cm} .
$$

Therefore, a prompt neutron with $1 \mathrm{MeV}$ energy should have its energy after it travels around $25 \mathrm{~cm}$,

$$
E_{n}^{\prime}=1 \mathrm{MeV} \times\left(\frac{1}{2}\right)^{25} \simeq 0.03 \mathrm{eV} .
$$

This neutron does not have to travel linearly, but in any case, it should become a thermal neutron.

\subsection{Mean Free Path of Thermal Neutron in the $n$-235U Fission Process}

We can easily calculate the mean free path of the thermal neutron before the nuclear fission in one batch solution. Since $\sigma_{f}=585 \mathrm{~b}$, we find

$$
\lambda_{f}=\frac{1}{\rho \sigma_{f}} \simeq 11 \mathrm{~cm}
$$

From these considerations, we see that prompt neutrons with $1 \mathrm{MeV}$ should travel around $25 \mathrm{~cm}$, and then they become thermal neutrons. Further, after they travel $11 \mathrm{~cm}$, they can induce nuclear fissions. Thus, if one carries $50 \ell$ of the uranium nitrate solution into the sedimentation tank with $45 \mathrm{~cm}$ diameter and $25 \mathrm{~cm}$ height, then nuclear chain reactions may well start quickly and proceed further on.

\subsection{Reaction Time of Neutrons}

Now we see that when prompt neutrons travel $36 \mathrm{~cm}$, then they can induce nuclear fissions. Therefore, we should estimate the duration time that is necessary to travel this $36 \mathrm{~cm}$. Since the nuclear reaction time must be smaller than $10^{-15}$ second, we can ignore this time duration. Since the prompt neutron with $1 \mathrm{MeV}$ should spend $\tau_{0} \simeq 7.6 \times 10^{-10}$ second to proceed $1 \mathrm{~cm}$, its energy becomes a half of the previous energy after $1 \mathrm{~cm}$ walk. Therefore, the time to proceed the next $1 \mathrm{~cm}$ becomes larger by a factor of $\sqrt{2}$. In this way, if the prompt neutron proceed $25 \mathrm{~cm}$, then the total time to spend must be

$$
T_{0}=\left(1+\sqrt{2}+\cdots+2^{25 / 2}\right) \tau_{0} \simeq 15 \mu \mathrm{s} .
$$

After that, this neutron becomes thermal, and it should proceed $11 \mathrm{~cm}$ before the nuclear fission. Since the thermal neutron may have the energy of $0.03 \mathrm{MeV}$, it should take $\tau_{\text {th }} \simeq 46 \mu \mathrm{s}$. Thus, the total time that is necessary for the prompt neutron to induce a fission reaction should be $T_{\text {tot }} \simeq 61 \mu \mathrm{s}$.

\section{Total Energy of Fission with Criticality}

Here, we should estimate the total amount of energy which is released from this accident. This evaluation must be very difficult, but we want to calculate it in an approximate way and obtain an order of magnitude of the total energy.

First, the number of neutrons which is required for the criticality reactions should be taken as $n_{r}=1.001$, which is assumed to be consistent with the total energy released as calculated from the computer simulation. In 
nuclear reactors, one should make use of all the possible techniques to keep the number as $n_{r}=1$.

In addition, we assume that the number of nuclear fissions should be $N=40000$. This number is chosen so that the total nuclear energy release should be consistent with the computer simulation which can reproduce all the observed radiation energies. In this case, the total reaction time of fission becomes $T_{f} \simeq 2.4 \mathrm{~s}$, and the total number of fissions becomes

$$
N_{\text {tot }}=1.001^{40000} \simeq 2.3 \times 10^{17} .
$$

Further, we evaluate the neutron number at the beginning, and this neutron should come from the spontaneous fission of ${ }^{238} \mathrm{U}$. The number of neutrons in one batch solution must be around 20 , and we take a half of this number. The energy release from the nuclear fission must be around $200 \mathrm{MeV}$ in each reaction, and therefore the total energy becomes

$$
E_{\text {tot }} \simeq 4.6 \times 10^{26} \mathrm{eV}
$$

which is just similar to the result of the computer simulation.

\section{Why Does the Criticality Stop?}

It is true that the criticality accident produced a huge amount of energy by the nuclear chain reactions, and the accident is indeed quite serious. In this sense, we here clarify as to how the chain reactions started and continued by reaching the critical stage. However, we face to the more serious problem at this point. That is, why the criticality accident could stop? We should understand any reason why the criticality could stop, namely there were only one burst and not any more burst, but why?

\subsection{Nuclear Fission in the Seventh Batch}

Here, we try to answer for this question, though it should be extremely difficult. In order to find a possible mechanism for the stopping of the criticality, we assume that the uranium compound should settle faster than any other compounds in the solution. Further, we assume that uranium should be settled within $20 \%$ height from the bottom of the sedimentation tank.

In this case, after the sixth batch, the uranium should be settled up to the $4.9 \mathrm{~cm}$ from the bottom. Thus, water should be found for $19.7 \mathrm{~cm}$ long in the sedimentation tank. By taking into account this fact, we can calculate the total energy release by nuclear fission as

$$
E_{\text {tot }} \simeq 4.6 \times 10^{26} \mathrm{eV} \simeq 7.4 \times 10^{7} \mathrm{~J} .
$$

The duration time of this nuclear reactions can be estimated and should be around $T_{f} \simeq 2.4 \mathrm{~s}$, which should correspond to the time that the uranium compound is coming down to the bottom.

\subsection{Nuclear Fission in the Sixth Batch}

The same calculation can be carried out for the sixth batch case. In this case, we see that the total energy must be 1000 times smaller than that of the seventh batch case. This is not very large, but at the sixth batch, the nuclear chain reactions already started, and indeed there were a small burst.

From this calculation, we now understand the reason why the criticality stopped. In case the uranium were settled at the bottom of the tank, then the nuclear chain reaction cannot proceed further since the prompt neutrons cannot lose their energy because of the lack of water.

\subsection{Nuclear Fission in the Eighth Batch}

From now on, we only present a possible scenario of nuclear accident, if the 8th batch were carried into the tank. In this case, the number of uranium involved in the nuclear fission must be proportional to the height of water, and thus it should be $\frac{22.9}{19.7}$ more than the seventh batch. Thus, the number becomes

$$
N=40000 \times \frac{22.9}{19.7} \simeq 46500 .
$$


This means that the number of nuclear fissions should be also increased and the total number becomes

$$
N_{\text {tot }}=1.001^{46500} \simeq 1.5 \times 10^{20} .
$$

Therefore, the total energy becomes

$$
E_{\text {tot }} \simeq 3 \times 10^{29} \mathrm{eV} \simeq 4.8 \times 10^{10} \mathrm{~J} .
$$

This energy $4.8 \times 10^{10} \mathrm{~J}$ corresponds to 11 ton of TNT powder which is quite a serious explosion. The accident of Chernobyl nuclear power plant is believed to correspond to around 100 ton of TNT powder, and therefore, if the 8th batch were thrown away, then the accident would have been more than serious.

\section{Summary}

We have discussed the basic mechanism of the JCO accident in terms of the nuclear multiple scattering theory. In this paper, we have clarified how the nuclear chain reactions could proceed in the small area of the sedimentation tank. The JCO accident should be studied from the point of view science, even though there must be no serious technical problems in nuclear reactors. In this respect, one may say that the JCO accident is rather similar to scientific phenomena, and it is essentially different from problems found in the nuclear power plants.

\section{Acknowledgements}

We are grateful to N. Yoshinaga for interesting discussions and useful comments.

\section{References}

[1] (2005) Nihon Genshiryoku Gakkai JCO Jiko-Chosa Iinkai, JCO Rinkaijiko; Sonozenbou no kaimei jijitsu youin taiou (Criticality Accident in JCO; Its Solution, Fact, Cause and Study). Tokai University Press, Kanagawa. [In Japanese]

[2] Yamane, Y., Nakajima, K., Abe, H., Hayashi, Y., Arisawa, J. and Hayami, S. (2010) Kakunenryo-shisetsu no Jiko-eikyou-hyoukashuhou ni kansuru chosa (V) Rinkaijiko-eikyo no hyouka-shuhou to Shikaiseki (Research on Consequence Analysis Method for Probabilistic Safety Assesment of Nuclear Fuel Facilities (V): Evaluation Method and Trial Evaluation of Criticality Accident). Atomic Energy Society of Japan, Tokyo, Vol. 9, No. 1, 96-107. [In Japanese]

[3] Tateno, J., Noguchi, K. and Aoyagi, N. (2000) Tetteikaimei Tokaimura Rinkaijiko (Criticality Accident in Tokaimura; Complete analysis). Shin-nihon Shuppan-sha, Tokyo. [In Japanese]

[4] Tonoike, K., Nakamura, T., Yamane, Y. and Miyoshi, Y. (2003) Power Profile Evaluation of the JCO Precipitation Vessel Based on the Record of the Gamma-Ray Monitor. Nuclear Technology, 143, 364.

[5] Yamane, Y., Nakajima, K., Yamamoto, T. and Miyoshi, Y. (2003) JAERI-Conf 2003-019, 740-745. Development of Criticality Accident Analysis CodeAGNES.

http://www.iaea.org/inis/collection/NCLCollectionStore/_Public/36/116/36116549.pdf http://jolissrch-inter.tokai-sc.jaea.go.jp/pdfdata/JAERI-Conf-2003-019-Part2.pdf

[6] Bohr, A. and Mottelson, B.R. (1998) Nuclear Structure, 1.

[7] Glauber, R.J. (1959) In: Brittin, W.E. and Dunham, L.G., Eds., Lectures in Theoretical Physics, Vol. 1, Interscience, New York, p. 315.

[8] Fujita, T. and Hüfner, J. (1979) Physics Letters, 87B, 327-331. http://dx.doi.org/10.1016/0370-2693(79)90546-X

[9] Fujita, T. and Hüfner, J. (1980) Nuclear Physics A, 343, 493-510. http://dx.doi.org/10.1016/0375-9474(80)90666-1

[10] Okajima, S., Kugo, T. and Mori, T. (2012) Genshiro Butsurigaku (Reactor Physics). Ohmsha, Tokyo. [In Japanese]

[11] Nuclear Data Center. http://wwwndc.jaea.go.jp/j40fig/jpeg/h001_f1.jpg 


\section{Submit or recommend next manuscript to SCIRP and we will provide best service for you:}

Accepting pre-submission inquiries through Email, Facebook, LinkedIn, Twitter, etc.

A wide selection of journals (inclusive of 9 subjects, more than 200 journals)

Providing 24-hour high-quality service

User-friendly online submission system

Fair and swift peer-review system

Efficient typesetting and proofreading procedure

Display of the result of downloads and visits, as well as the number of cited articles

Maximum dissemination of your research work

Submit your manuscript at: http://papersubmission.scirp.org/ 